\title{
Segmentation of Multi-Textured Images using Optimized Local Ternary Patterns
}

\author{
G. Madasamy Raja \\ Associate Professor, \\ Department of Information Technology, \\ PET Engineering College, Vallioor, \\ Tamilnadu -627117, India
}

\author{
V. Sadasivam, Ph.D \\ Principal, \\ PSN College of Engineering \& Technology, \\ Tirunelveli, \\ Tamilnadu - 627152, India
}

\begin{abstract}
Texture segmentation is a process of segmenting an image into differently textured regions. This paper is aimed at the segmentation of multitextured images by using Optimized Local Ternary Patterns (OLTP), a new texture model which is recently proposed for texture analysis. This paper uses unsupervised texture segmentation method with the application of optimized local ternary patterns for finding the dissimilarity of adjacent image regions during the segmentation process. The performance of this recently proposed texture measure OLTP is evaluated, compared with other texture models Texture Spectrum (TS) and Local Binary Patterns (LBP) and found to be the best.
\end{abstract}

\section{Keywords}

Texture Spectrum, Texture Segmentation, Kullback-Leibler Distance, Local Binary Patterns, Optimized Local Ternary Patterns, Brodatz Textures.

\section{INTRODUCTION}

Image segmentation is a useful tool in many applications for identifying regions of interest (ROI) in a digital image [1]. Undoubtedly texture is one of the principal features used in image processing and pattern recognition [2]. Texture plays an important role in many machine vision tasks such as surface inspection, scene classification, surface orientation, and shape determination. Texture can be characterized by the spatial distribution of image pixel values in a neighborhood region. Though texture is widely used it has no precise definition due to its wide variability [3]. Since a digital image is made up of pixels, texture can be defined as an entity consisting of mutually related group of pixels. This group of pixels is called as texture primitives or texture elements or texels [4].

Texture analysis is one of the most important techniques used in the investigation and interpretation of images that consists of repetition or quasi repetition of some fundamental image elements [5]. Texture classification and Texture segmentation are the primary issues in texture analysis. In texture classification, a given texture region is identified from a given set of texture classes using various texture features. Unlike texture classification, texture segmentation determines the boundaries between various textured regions in an image [6]. Texture segmentation is a process which accurately partitions an image into several distinct textured regions.

The segmentation can be either supervised or unsupervised. Unsupervised segmentation is a very challenging research problem in which only limited success has been achieved so far because no prior information about the textures present in the image is available. Usually, one does not know what types of textures exist in an image, how many textures there are, and what regions contain which textures [7]. Some examples for unsupervised texture segmentation methods are split-andmerge technique [8], quad tree method that combines statistical information with spatial information [9], and texture spectrum technique [10]. Unsupervised segmentation of images containing texture primitives at very different scales is not viable, because it is hard to discriminate small image regions from large texture primitives without any prior knowledge [11].

In supervised segmentation, prior knowledge of the type of textures present in the image is available. Texture segmentation with stochastic optimization [12] and Texture segmentation using optimized separation [13] are some examples in the literature for supervised segmentation. For any textured analysis, the main conceptual difficulties are to define a texture measure and a texture classification method [14].

There are two categories for texture measures and they are Model based and Non-Model based texture measures. Model based texture measures construct an image model which is based on the original image to describe the texture. Gibbs Markov Random Field (GMRF) [15] and Auto Regressive model [16] are some examples for model based texture measurement. Markov Random Field assumes that the intensity at each pixel in the image depends on the intensities of only neighboring pixels whereas Auto Regressive model approximates a pixel in an image as the linear combination of its local neighbors.

Statistical, Structural and Transform based methods are few methods which are coming under the category of Non-Model based texture measures. Statistical methods deals with first order and second order statistics of image pixels where as Structural methods describe textures as combination of well defined texture primitives that are placed according to some syntactic rules. Transform based methods represent an image in a new form so that the texture characteristics of texture can be more easily accessed. Fan and Xia [17] used the relationship between the periodicity of natural textures and subbands of wavelet transforms. Colour contrast was used in the segmentation methods of both Chen et al. [18] and Chang et al. [19]. By using a color gradient detection technique, a new color image segmentation algorithm was proposed by Luis Ugarriza et al. [20]. Uniform Extended Local Ternary Patterns by Wen-Hung Liao and Young [21], Ternary Pattern Operator by Suguna and Anandhakumar [22] and Compound Local Binary Pattern (CLBP) by Faisal Ahmed et al. [23] are 
some recent developments in the textural analysis under NonModel based texture measures.

Texture classification results obtained by using Optimized Local Ternary Patterns (OLTP), a recent study by Madasamy Raja and Sadasivam [24], have already been proved better than those obtained with the existing methods. This OLTP texture model may also be used for texture segmentation of multitextured images. The aim of this paper is to further evaluate the performance of OLTP in the discrimination of textures that are available in multitextured images and demonstrate its usefulness for texture segmentation.

This paper is organized as follows: Section 2 describes already existing texture measures Texture Spectrum (TS) and Local Binary Patterns (LBP). In this section, the newly proposed texture model Optimized Local Ternary Patterns (OLTP) is also briefly reviewed. Segmentation algorithm which is common to all the experiments in this paper is illustrated in Section 3. Experimental results are then discussed in section 4 and conclusions are given in section 5 .

\section{TEXTURE MEASURES}

This section gives a brief review of computationally efficient texture feature extraction methods namely Texture Spectrum (TS), Local Binary Patterns (LBP) and Optimized Local Ternary Patterns (OLTP).

\subsection{Texture Spectrum (TS)}

In this method, the local texture information is extracted by comparing the central pixel $\left(\mathrm{g}_{\mathrm{c}}\right)$ of a $3 \times 3$ local neighborhood with its 8 neighbors $\left(\mathrm{g}_{\mathrm{i}}, \mathrm{i}=0,1,2, \ldots, 8\right)$. This $3 \times 3$ neighborhood region constitutes the smallest unit called "Texture Unit (TU)" that contains the comparison results of eight surrounding neighbors with the center pixel. In a $3 \times 3$ neighborhood region, there will be 8 such comparison results (E1, E2, E3, E4, E5, E6, E7, E8)

$$
\mathrm{E}_{\mathrm{i}}=\left\{\begin{array}{lll}
0 & \text { if } & \mathrm{g}_{\mathrm{i}}<\mathrm{g}_{\mathrm{c}} \\
1 & \text { if } & \mathrm{g}_{\mathrm{i}}=\mathrm{g}_{\mathrm{c}} \\
2 & \text { if } & \mathrm{g}_{\mathrm{i}}>\mathrm{g}_{\mathrm{c}}
\end{array} \quad \text { for } \mathrm{i}=1,2,3, \ldots 8\right.
$$

As such element of TU has one of three possible values ( 0 or 1 or 2), TU ranges from 0 to 6560 and in total there are 6561 texture units $\left(3^{8}\right)$. The histogram of these texture units forms a 'Texture Spectrum' that represents the image texture description and it can be used as a texture measure for texture analysis. The main disadvantage of this method is its complexity due to large number of texture units that ranges from 0 to 6561 .

\subsection{Local Binary Patterns (LBP)}

The texture model Local Binary Patterns (LBP) was originally introduced by Ojala et al. [25] and it was really a very powerful method for texture representation. LBP has been used successfully for a variety of pattern recognition tasks. It considers a $3 \times 3$ neighborhood and uses the value of the center pixel of that neighborhood as a threshold, to label the pixels of the neighborhood region. All these 8 labeled values form a binary sequence which is considered as a pattern string.

For a $3 \times 3$ neighborhood around a centre pixel in an image, the LBP operator is defined as

$$
\begin{gathered}
\mathrm{LBP}_{8}=\sum_{\mathrm{n}=1}^{8} 2^{\mathrm{n}} \mathrm{S}\left(\mathrm{i}_{\mathrm{n}}-\mathrm{i}_{\mathrm{c}}\right) \\
\text { and } \\
\mathrm{S}(\mathrm{U})= \begin{cases}1 & \text { if } \mathrm{u} \geq 0, \\
0 & \text { otherwise }\end{cases}
\end{gathered}
$$

where $\mathrm{n}$ is the number of pixels in the considered neighborhood, $i_{c}$ is the pixel value of center pixel $c$ and $i_{n}$ are the pixel values of neighborhood considered. So, for a $3 \times 3$ neighborhood a total of $256\left(2^{8}\right)$ different pattern strings can be generated. An entire image can be represented by a pattern histogram of 256 elements, which can be used as the texture descriptor.

In LBP, when larger neighborhoods are used, correspondingly, the number of pattern strings will get increased. When the subset of pattern strings is considered, the length of the pattern histogram is reduced. With the introduction of the concept of uniform patterns, the length of pattern histogram was further reduced without significant loss in its discrimination ability. As the correlation between pixels in a small neighborhood region is high, LBP efficiently represents the texture information in a digital image. LBP operator is invariant to any gray scale transformation. In the limitation point of view, LBP supports only a binary level comparison for encoding and so it is inadequate to represent the local texture information in more detail. Moreover it is sensitive to noise also.

\subsection{Optimized Local Ternary Patterns (OLTP)}

In OLTP, a texture image can be decomposed into a set of small units called patterns. As the texture model OLTP uses only optimal patterns (selected number of uniform patterns), the length of the pattern histogram is maintained as optimum.

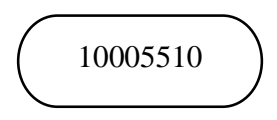

(a)

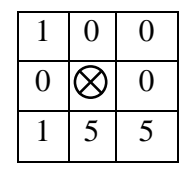

(b)

\begin{tabular}{|l|l|l|}
\hline 252 & 172 & 168 \\
\hline 156 & 256 & 108 \\
\hline 244 & 380 & 332 \\
\hline
\end{tabular}

(c)
Figure 1: Example of transforming a 3x3 neighborhood to a pattern string in OLTP texture model. (a) $3 \times 3$ Local Region (b) Pattern Matrix (c) Pattern String

In OLTP, a pattern is represented by eight elements, each of which has one of three possible values $(0,1,5)$ that are obtained from a neighborhood of $3 \times 3$ pixels. Let $i_{c}, i_{1}, i_{2}$, $\ldots, i_{8}$ be the pixel values of a local $3 \times 3$ neighborhood region, where $i_{c}$ is the value of the central pixel and $i_{1}, i_{2}, \ldots, i_{8}$ are the pixel values of its 8 neighbors. The following equation (3) defines the process for converting a local $3 \times 3$ neighborhood into its pattern representation $(\mathrm{P})$,

$$
P\left(i_{c}, i_{n}\right)=\left\{\begin{array}{lll}
0 & \text { if } & i_{n}<(1-\sigma) i_{c} \\
1 & \text { if } & (1-\sigma) i_{c} \leq i_{n} \leq(1+\sigma) i_{c} \\
5 & \text { if } & i_{n}>(1+\sigma) i_{c}
\end{array}\right.
$$


where $\sigma$ is a small scaling factor and it is assigned as 0.05 . Figure 1 shows an example of transforming a sample $3 \times 3$ local neighborhood into a pattern unit by using equation (3). For a $3 \times 3$ local neighborhood region, the total number of different pattern strings will be $6561\left(3^{8}\right)$. In other words, Eq. 3 will deliver any one pattern string from a set of 6561 different pattern strings for a $3 \times 3$ local neighborhood region when OLTP texture model is used. So, by using this OLTP texture model a complete texture image can be described by a pattern histogram of 6561 bins that represents the occurrence frequency of pattern strings over the texture image.

It is observed that for a $3 \times 3$ local neighborhood of a texture image, only few pattern strings among these 6561 different pattern strings, are frequently occurring patterns and all other pattern strings are not so. So it is meaningless to allot separate bins for all the pattern strings because it may end in wastage of memory and wastage of time. Further, the analysis of these frequently occurring pattern strings found that all these pattern strings have uniform circular structure and they are rotation invariant. To identify these frequently occurring pattern strings which are rotation invariant, let us consider, a uniformity measure ' $U$ ' which corresponds to the number of spatial transitions circularly among the sub patterns in the pattern strings. The uniformity measure ' $U$ ' is defined as

$$
\begin{aligned}
& U=F\left(P\left(i_{c}, i_{1}\right), P\left(i_{c}, i_{8}\right)\right)+\sum_{n=2}^{8} F\left(P\left(i_{c}, i_{n}\right), P\left(i_{c}, i_{n-1}\right)\right) \\
& \text { where } F(A, B)= \begin{cases}1 & \text { if }|A-B|>0 \\
0 & \text { otherwise }\end{cases}
\end{aligned}
$$

For example, the pattern string 11111111 has $U$ value of 0 , the pattern string 00000055 has $U$ value of 2 and the pattern string 55501111 has $U$ value of 3 . Patterns with $U$ value of less than or equal to three are considered as "Uniform Pattern Strings" and totally 45 uniform pattern strings are available. The implementation of a new idea to calculate the Transition Length $(\rho)$ among the uniform patterns and the introduction of a new concept called Level of Optimality $\left(\mathrm{L}_{\mathrm{opt}}\right)$ have paved the way to select the subset of uniform patterns which are designated as optimal patterns. The elements of the uniform pattern strings are either 0 or 1 or 5 or any combinations of these values. If the number of occurrences (cardinality) of 0 , 1 and 5 are represented by $\operatorname{Card}(0), \operatorname{Card}(1)$ and $\operatorname{Card}(5)$ respectively, then the level of optimality for a particular uniform pattern string can be computed as,

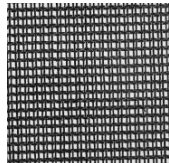

(a)

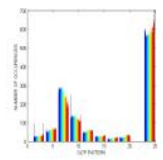

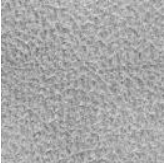

(b)

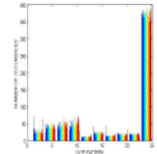

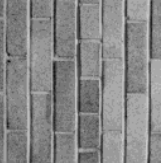

(c)

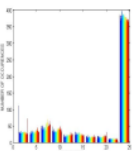

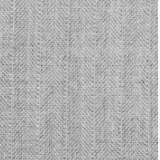

(d)
Figure 2: Selected texture images and their corresponding pattern spectrum obtained through OLTP texture model. (a) French Canvas (b) Pigskin (c) Brick (d) Herringbone Weave $(e),(f),(g),(h)$ corresponding pattern spectrum of the images in figures $2(\mathrm{a}),(\mathrm{b}),(\mathrm{c}),(\mathrm{d})$.

$$
\mathrm{L}_{\mathrm{opt}}=\frac{1+\min \{\operatorname{Card}(0), \operatorname{Card}(1), \operatorname{Card}(5)\}}{\max (\rho)}
$$

where $\rho$ refers to the transition length among the sub patterns in the uniform pattern strings. Table 1 contains the transition lengths of various sub patterns that are available in the uniform pattern strings.

Optimized Local Ternary Patterns (OLTP) texture model that uses only optimal set of uniform patterns to represent a local image texture is defined as:

$\operatorname{OLTP}= \begin{cases}\sum_{\mathrm{i}_{\mathrm{c}}=1}^{8} \mathrm{P}\left(\mathrm{i}_{\mathrm{c}}, \mathrm{i}_{\mathrm{n}}\right) & \text { if } \mathrm{U} \leq 3 \text { and } \mathrm{L}_{\mathrm{opt}} \geq 2 \\ 25 & \text { otherwise }\end{cases}$

This recently proposed texture model, OLTP uses only 24 unique optimal patterns for texture representation and it groups all other patterns under one label 25 which are termed as "sub-optimal" patterns. Therefore the dimension of pattern spectrum has been reduced from 6561 to 25 , that too with optimal set of patterns. The occurrence frequency of all the optimal patterns over a whole image is termed as "Pattern Spectrum" or "Pattern Histogram", which reflects the texture information of that image.

Textures have so many different dimensions and obviously a texture model should show different pattern spectrum for different texture images. OLTP texture model delivers different pattern spectrum for different texture images which is successfully proved in Figure 2. Figure 2(a), 2(b), 2(c) and 2(d) show some of the selected texture images from Brodatz album [26] and Figure 2(e), 2(f), 2(g) and 2(h) show their corresponding pattern spectrum of the optimal patterns obtained through OLTP texture model. 
Table 1: Details of the Transition Length ( $\rho$ ) for the sub patterns of uniform pattern strings

\begin{tabular}{|c|c|c|}
\hline \multicolumn{3}{|c|}{ Transition Length } \\
\hline S.No & Sub Pattern type & $\begin{array}{l}\text { Transition } \\
\text { Length }(\rho)\end{array}$ \\
\hline 1 & '01' & 1 \\
\hline 2 & '10' & 1 \\
\hline 3 & ' 15 ' & 1 \\
\hline 4 & ‘51' & 1 \\
\hline 5 & '05' & 2 \\
\hline 6 & ‘50’ & 2 \\
\hline 7 & '015' & 1 \\
\hline 8 & ‘510’ & 1 \\
\hline 9 & ' 105 ' & 2 \\
\hline 10 & $' 150 ’$ & 2 \\
\hline 11 & '051' & 2 \\
\hline 12 & '501' & 2 \\
\hline
\end{tabular}

OLTP texture method was empirically proven successfully in texture classification for a wide range of textures in the previous study under different kinds of experimental settings like gray-scale variation, rotation variation, histogram equalization and noise. In this study, the segmentation performance of the recently developed OLTP texture model for a variety of multitextured images is pursued.

\section{TEXTURE SEGMENTATION}

\subsection{Texture Segmentation Principle}

This study is considered as a test bench to demonstrate the discrimination ability of the newly developed OLTP texture model. The evaluation process uses a supervised segmentation approach over the selected texture images which are extracted from the standard Brodatz texture database. The steps involved in the process of texture segmentation are shown in Figure 3.

\subsection{Texture Segmentation Algorithm}

The algorithm of the texture segmentation is described as follows.

Step 1. Read the texture mosaic image.

Step 2. Select randomly a sample sub image of 30x30 pixels from the texture image as sample image.

Step 3. Transfer the $30 \times 30$ sub image into the OLTP texture model and obtain the OLTP spectrum for the selected sub image.
Step 4. Select one sample sub image for each selected texture image and repeat step 1 to step 3 for all the selected texture sub images (because this study follows supervised segmentation)

Step 5. Select a window size of 30x30 pixels starting from the top-left corner of the test image and do step 3 for the selected window.

Step 6. Calculate Kullback-Leibler distance (D) between the OLTP spectrum of the selected window obtained from step 5 and one of every sample obtained from step 4.

Step 7. The central pixel of the window considered in step5 will be assigned to a particular texture class of the sample, for which the D (obtained from step 6) is the minimum among all the samples.

Step 8. Repeat step 5 to step 7 for all the windows which are selected in size 30x30 pixels starting from the top-left corner of the given test image with a step of two pixels in the row and column directions.

\subsection{Kullback-Leibler Distance Measure}

Similarity is a quantity that reflects the strength of relationship between two objects or two features. Distance measures dissimilarity. Dissimilarity measures the discrepancy between the two objects on several features. There are many types of distance measures and each distance measure has its own characteristics. The Kullback-Leibler distance (D) between two distributions $s$ and $m$ is defined as

$\mathrm{D}(\mathrm{s}: \mathrm{m})=\sum_{\mathrm{i}=1}^{\mathrm{n}} \mathrm{s}_{\mathrm{i}} \log \left(\mathrm{s}_{\mathrm{i}} / \mathrm{m}_{\mathrm{i}}\right)$

where $s$ and $m$ are the sample and model distributions, $n$ are the number of bins and $s_{i}, m_{i}$ are the respective sample and model probabilities at a particular bin $i$.

The modified version of the same Kullback-Leibler distance (G) is

$$
G=2\left(\left[\sum_{s, m} \sum_{i=1}^{n} f_{i} \log f_{i}\right]-\left[\sum_{s, m}\left(\sum_{i=1}^{n} f_{i}\right) \log \left(\sum_{i=1}^{n} f_{i}\right)\right]\right.
$$$$
\left.-\left[\sum_{\mathrm{i}=1}^{\mathrm{n}}\left(\sum_{\mathrm{s}, \mathrm{m}} \mathrm{f}_{\mathrm{i}}\right) \log \left(\sum \mathrm{f}_{\mathrm{i}}\right)\right]+\left[\left(\sum_{\mathrm{s}, \mathrm{m}} \sum_{\mathrm{i}=1}^{\mathrm{n}} \mathrm{f}_{\mathrm{i}}\right) \log \left(\sum_{\mathrm{s}, \mathrm{m}} \sum_{\mathrm{i}=1}^{\mathrm{n}} \mathrm{f}_{\mathrm{i}}\right)\right]\right)
$$

where $s$ and $m$ are the sample and model distributions, $n$ is the number of bins and $s_{i}, m_{i}$ are the respective sample and model probabilities at a particular bin $i$. In this study, the modified version of Kullback-Leibler distance is used to find the similarity between two OLTP spectrums of test image window and sample sub images. 


\section{EXPERIMENT RESULTS AND DISCUSSION}

\subsection{Image data}

The texture segmentation problem deals with the image that contains several different types of texture (composite texture image) and segments that image according to the textures available in it. More than 400 composite texture images of 256 gray levels are created using the selected textures from Brodatz album texture images. This study tests the texture segmentation ability of the Optimized Local Ternary Patterns (OLTP) texture approach by performing supervised segmentation on several composite test images of varying complexity, which are shown in Figure 4.

\subsection{Experimental Results}

Figure 5 shows some example segmentation results when OLTP texture model was used in the segmentation algorithm that was discussed in the previous section for various composite texture mosaic images stitched from the selected texture images of Figure 4. The left column of Figure 5(Figure 5(a)-(f)) show the test images that are involved in the experiments and the results of the segmentation are shown in the right column of Figure 5(Figure 5(g)-(1)). The segmentation results are displayed as grey level images, where regions belonging to different textures are shown with different grey levels. All the results in Figure 5 (Figure 5(g)(1)) consist of correctly identified number of textures as well as good segmentation.

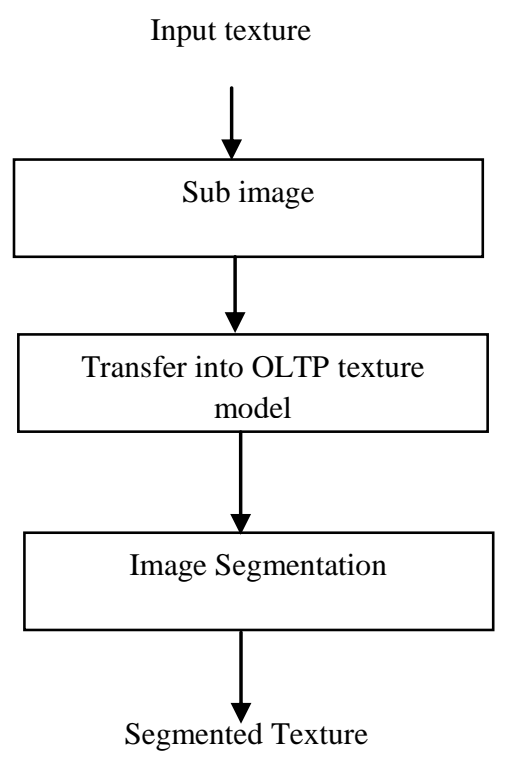

Figure 3: Texture segmentation procedure.

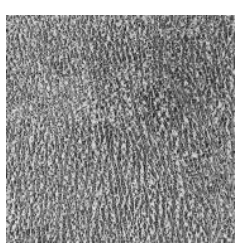

(a)

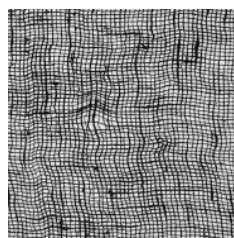

(d)

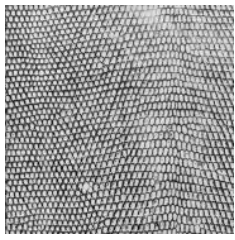

(g)

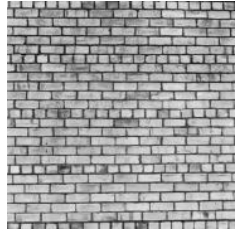

(b)

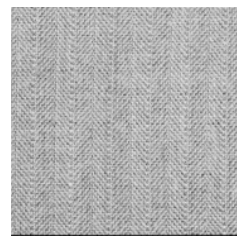

(e)

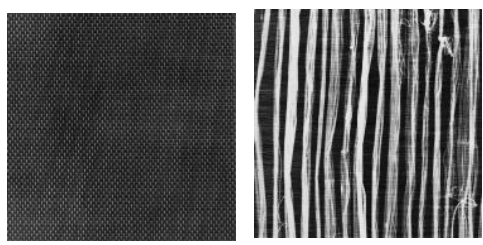

(h)

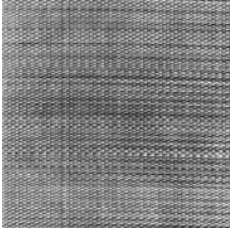

(c)

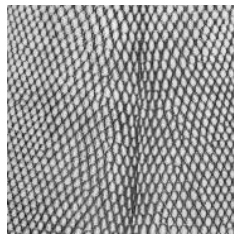

(f)

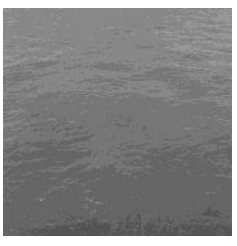

(j)

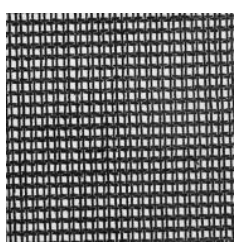

(k)
Figure 4: The Brodatz texture images used in the segmentation experiments
(a) ) pressed calf leather
(b) brick wall
(c) straw matting
(d) loose burlap
(e) woven aluminium wire
(f) lizard skin
(g) reptile skin
(i) raffia
(h) herringbone weave
(k) french canvas
(j) water

(i) 


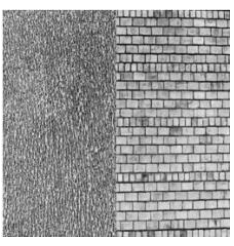

(a)

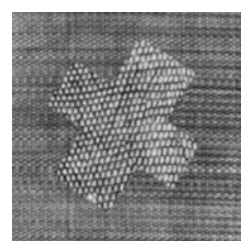

(b)

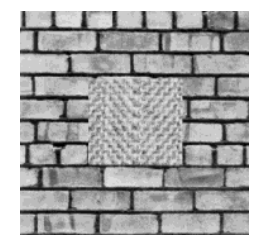

(c)

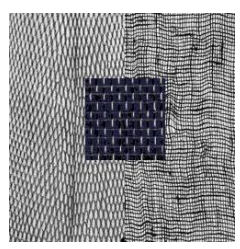

(d)

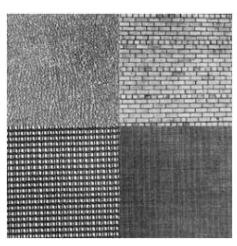

(e)

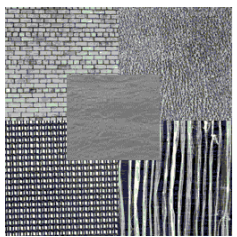

(f)

Figure 5: Supervised texture segmentation results when OLTP texture model is used. $5(a)-5(f)$ various multitextured images created from the selected texture images and $5(g)-5(I)$ shows the corresponding segmented output when OLTP texture model is used.

\subsection{Result Analysis}

The stitched two texture mosaic image of Figure 5(a) consists of textures pressed calf leather and brick wall. The twotexture mosaic image of Figure 5(b) consists of textures reptile skin and straw matting. The two-texture mosaic image of Figure 5(c) consists of textures herring bone weave and brick wall. Result images Figure 5(g), 5(h) and 5(i) are showing the segmentation results of input images Figure 5(a), 5(b) and 5(c) respectively. In all these result images, the OLTP texture model successfully discriminates the two textured regions and detects the boundary between them quite clearly. The three-texture mosaic image Figure 5(d) consists of textures woven aluminium wire, lizard skin and loose burlap. The result image Figure 5(j) for this input image contains correctly identified number of textures as well as good segmentation. The stitched four-texture mosaic image Figure 5(e) consists of textures herring bone weave, french canvas, pressed calf leather and brick wall. The five-texture mosaic image Figure 5(f) consists of textures raffia, french canvas, pressed calf leather, water and brick wall. These input texture mosaic images are difficult images to discriminate for several approaches but OLTP texture model gives a reasonably good segmentation results (Figure $5(\mathrm{k})$ and Figure $5(1))$ with some negligible errors around texture boundaries.

The lack of suitable quantitative measures for finding the accuracy of segmentation result makes it very difficult to evaluate and compare texture segmentation algorithms. One simple measure that is widely used is the percentage of misclassified pixels. Table 2 gives the percentage of misclassified pixels for the segmentation experiments that are conducted for 100 test composite texture mosaic images. All the images give an error percentage of below $4.5 \%$ which can be considered as a good result. It is known that when more texture boundaries are there in an image, then more difficult decisions must be made which may result in an increasing number of misclassified pixels.

Appreciably, from these results, it is also observed that when the newly proposed OLTP texture model is used even boundary pixels are also segmented properly. To demonstrate the benefit of the newly proposed texture descriptor OLTP, in differentiating textures, a comparative experiment has also been carried out. This experiment uses 50 randomly chosen test images with same sizes from the already selected multitextured image set irrelevant of the number of textures available in the mosaic images.

Table 3 compares the error percentage of the incorrectly classified pixels obtained by applying different segmentation methods to each of 50 randomly chosen test images. The averaged errors over the entire test images for this comparative experiment are listed in Table 3. The right column illustrates the results of the newly proposed OLTP texture method, where as the two middle columns show the results obtained by applying the Texture Spectrum (TS) method and Local Binary Pattern (LBP) method.

The newly proposed OLTP texture model shows the best discrimination ability by achieving the least overall segmentation error. Both Texture Spectrum (TS) and Local Binary Pattern (LBP) methods were never found to perform best. It is also observed that both TS and LBP methods failed to locate accurate boundaries separating the regions in many test images. The newly proposed texture model OLTP, outperforms the other popular texture models Texture Spectrum (TS) and Local Binary Pattern (LBP) by achieving 
smaller segmentation error and this shows the reliability of this OLTP in texture analysis.

In segmentation problems, the window size refers to the size of the portion of images which are to be labeled. In order to analyze the effect of the window size of the sample test image when the newly proposed OLTP texture model is used, various sample sizes of $30 \times 30,20 \times 20,10 \times 10$ are considered for this experiment and the segmentation algorithm was applied to the randomly selected 25 composite texture mosaic images. These testing images are arbitrarily selected from the set of already created 400 multi-textured mosaic images, irrelevant of the number of textures available in a single mosaic image. Figure 6 gives the performance comparisons in terms of averaged segmentation accuracy among different texture methods for various sample window sizes. Here also both Texture Spectrum (TS) method and Local Binary Pattern (LBP) methods are considered along with the newly proposed texture model OLTP.

When the TS model is used, lowest average segmentation accuracy was obtained in all the three window sizes because for some texture images this method does not reveal the human perception of homogeneity. The reason is even if the human eyes see two neighboring pixels are equal in every aspect in the image rarely they have exactly the same intensity value. In homogeneous images, there should be lot of ones in the texture units, as the human eye perceives. However in reality, there is lack of ones when the TS model is used. If there is a lack of ones in the texture units, then TU will have only values of 0 and 2 , which may reduce the number of different texture units from $3^{8}$ to $2^{8}$. Obviously the length of the texture spectrum will be 256 instead of 6561 and hence this spectrum will never represent the real texture information that is available in the texture images. Another disadvantage in using TS model is the number of bins required for the histogram that is 6561 which is a huge value. When the length of the texture spectrum is high, then the computational time will also be high. Since the computational time for this model is pretty large, it cannot be used for the applications where computational time is a decisive factor. Moreover, texture spectrum method is sensitive to directional aspects of texture. These are some valid reasons for this texture method to perform poorly in the texture segmentation process.

The average segmentation accuracy of LBP model is better than Texture Spectrum model. LBP model is invariant against gray scale variations and rotational variations and hence it is suitable for real time applications. In LBP texture model, the computational complexity is very much reduced, since it uses only 10 bins in the pattern histogram. But it is practically not possible to characterize the local region by using merely 10 patterns, which is a drawback of this model and further LBP model is sensitive to noise also.

Among the three texture models, Texture Spectrum (TS) method uses ternary level comparison but uses large number of histogram bins and at the same time Local Binary Pattern (LBP) method uses only binary level comparison but uses limited number of histogram bins. The proposed OLTP method combines the advantages of both Texture Spectrum and Local Binary Pattern methods. In other words, the newly proposed OLTP method uses the ternary level comparisons for pattern representation like TS model and uses only less number of patterns like LBP model. From all the above results, it is justified that, the OLTP method gives superior performance by achieving the best segmentation accuracy to prove its discrimination ability. But one common point to be noted here is the larger window size results in better segmentation accuracy and this is proved in every texture models.

Table 2: Average error percentage of incorrectly classified pixels

\begin{tabular}{ccc}
\hline \hline $\begin{array}{c}\text { Number of } \\
\text { textures in an } \\
\text { image }\end{array}$ & $\begin{array}{c}\text { Number of } \\
\text { images } \\
\text { tested }\end{array}$ & $\begin{array}{c}\text { Percentage } \\
\text { of error }\end{array}$ \\
\hline 2 & 33 & 1.22 \\
3 & 30 & 2.56 \\
4 & 26 & 2.62 \\
5 & 11 & 4.12 \\
\hline \hline
\end{tabular}

Table 3: Comparison of segmentation performance

\begin{tabular}{lccc}
\hline \hline & & LBP & OLTP \\
Methods & TS & & \\
\hline $\begin{array}{c}\text { Averaged } \\
\text { Errors }\end{array}$ & 10.4 & 8.6 & 4.2 \\
\hline \hline
\end{tabular}

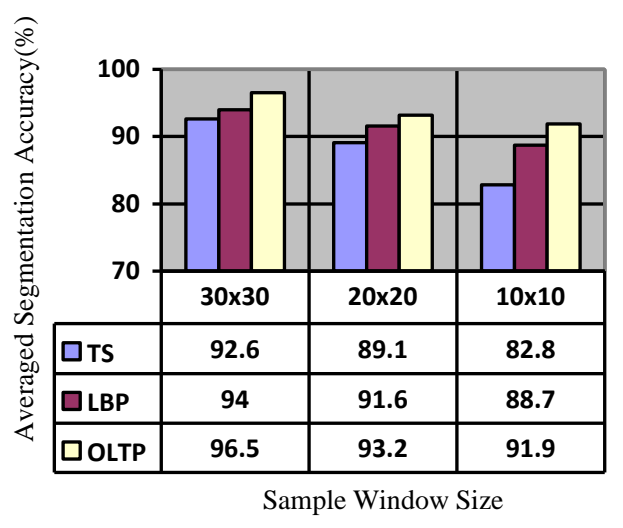

Figure 6: Comparison of segmentation accuracy among various texture models for various window sizes

\section{CONCLUSION}

In this study, the newly proposed texture model OLTP, has been evaluated from the point of view of texture segmentation performance. A simple segmentation algorithm has been used with this newly proposed texture model for segmenting the composite texture images made up of standard Brodatz texture images and promising results have been obtained in all the experiments. It is found that, for multi-textured images, the most robust segmentation performance was achieved by newly proposed OLTP texture model than the two popular texture models, Texture Spectrum (TS) and Local Binary Pattern (LBP) techniques.

This work uses Kullback-Leibler distance measure to evaluate the performance of the newly proposed texture model OLTP. As a future work, OLTP texture model can be used with other established distance measures to study the role of these measures in the segmentation accuracy and this may help in finding the best similarity measurement match for this newly 
proposed texture model OLTP. Only gray scale images have been used in this study and this work can be further extended for color images also. Another very useful direction for future research is this newly proposed OLTP texture model can be further tested with other useful image processing applications which are based upon texture retrieval.

Despite its computational simplicity, with its own minimal number of optimal set of patterns, the newly proposed texture model OLTP produced better and accurate results with reduced processing time.

\section{REFERENCES}

[1] S. Kothainachiar and R.S.D. Wahita Banu, "A novel image segmentation based on a combination of color and texture features," GVIP Journal, vol. 7, no. 2, Aug. 2007, pp. $45-51$.

[2] Dong Chen He and Li Wang, "Texture classification using texture spectrum," Pattern Recognition, vol. 23, no. 8, 1990, pp. $905-910$

[3] G.N. Srinivasan and G. Shobha, "Statistical Texture Analysis," in Proc. World Academy Of Science, Engineering And Technology, 2008, vol. 36, pp. 12641269.

[4] S. Arivazhagan and L. Ganesan, "Texture segmentation using wavelet transform," Pattern Recognition Letters, vol. 24, no. 16, Dec. 2003, pp. 3197-3203.

[5] P.P. Raghu and B. Yegnanarayana, "Segmentation of Gabor filtered textures using deterministic relaxation," IEEE Transactions on Image Processing, vol. 5, no. 12, Dec. 1996, pp. 1625-1636.

[6] R. Jain, Kasturi and B.G. Schunch, Machine Vision. McGraw Hill, 1995, pp. 234-240.

[7] M. Tuceryan and A. K. Jain, "Texture Analysis," in The Handbook of Pattern Recognition and Computer Vision, chapter 2.1. C. H. Chen, L. F. Pau and P. S. P. Wang, Ed. Singapore: World Scientific Publishing Co., 1993, pp. 235-276.

[8] P.C. Chen and T. Pavlidis, "Segmentation by texture using a co-occurrence matrix and a split-and-merge algorithm," Computer Graphics and Image Processing, vol. 10, no. 2, 1979, pp. 172-182.

[9] M. Spann and R. Wilson, "A quad-tree approach to image segmentation which combines statistical and spatial information," Pattern Recognition, vol. 18, no. 3/4, 1985, pp. 257-269.

[10] D.C. He and L. Wang, "Unsupervised textural classification of images using the Texture Spectrum," Pattern Recognition, vol. 25, no. 3, Mar. 1992, pp. 247255.

[11] T. Ojala and M. Pietikainen, "Unsupervised texture segmentation using feature distributions," Pattern recognition, vol. 32, no. 3, Mar. 1999, pp. 477-486.

[12] D.M. Tsai, S.K. Wu and M.C. Chen, "Optimal Gabor filter design for texture segmentation using stochastic optimization," Image and Vision computing, vol. 19, no. 5, Apr. 2001, pp. 299-316.

[13] T. Randen and J.H. Husoy, "Texture segmentation using filters with optimized energy separation," IEEE transactions on Image Processing, vol. 8, no. 4, Apr. 1999, pp. 571-582.
[14] L. Ma, L.P. Lu and L. Zhu, "Unsupervised texture segmentation based on multi-scale Local Binary Patterns and FCMs clustering," in IET International Conference on Wireless, Mobile and Multimedia Networks Proceedings (ICWMMN 2006), Hangzhou, China, pp. 14.

[15] K.H. Kim, B.S. Sharif and E.G. Chester, "Unsupervised texture analysis using a robust stochastic image model," in Proc.1997 6th International Conference on Image Processing and its Applications, Dublin, vol. 2, pp. 613 617.

[16] J. Mao and A. K. Jain, "Texture classification and segmentation using multiresolution simultaneous autoregressive models," Pattern Recognition, vol. 25, no. 2, Feb. 1992, pp. 173-188.

[17] G. Fan and X. Xia, "Wavelet-based texture analysis and synthesis using hidden Markov model," IEEE Transactions on Circuits and Systems I: Fundamental Theory and Applications, vol. 50, no. 1, 2003, pp. 106120.

[18] H.C. Chen, W.J. Chien and S.J. Wang, "Contrast-based color image segmentation," IEEE Signal Processing Letters, vol. 11, no. 7, July 2004, pp. 641-644.

[19] Y. Chang, D. Lee and Y. Wang, "Color-texture segmentation of medical images based on local contrast information," in Proc. IEEE Symposium on Computational Intelligence and Bioinformatics and Computational Biology, Honolulu, HI, 2007, pp. 488493.

[20] L. Garcia Ugarriza, E. Saber, S. R. Vantaram, V. Amuso, M. Shaw and R. Bhaskar, "Automatic image segmentation by Dynamic Region Growth and Multiresolution Merging," IEEE Transactions on Image Processing, vol. 18 , no. 10, Oct. 2009, pp. 2275-2288.

[21] W.H. Liao and T.J. Young, "Texture classification using Uniform Extended Local Ternary Patterns," in $12^{\text {th }}$ IEEE International Symposium on Multimedia(ISM 2010), Taiwan, pp. 191-195.

[22] R. Suguna and P. Anandhakumar, "A rotation invariant pattern operator for texture characterization," International Journal of Computer Science and Network Security (IJCSNS), vol. 10, no. 4, Apr. 2010, pp. 120129.

[23] F. Ahmed, E. Hossain, A. S. M. H. Bari and M. S. Hossen, "Compound Local Binary Pattern (CLBP) for rotation invariant texture classification," International Journal of Computer Applications (IJCA), vol. 33, no. 6, Nov. 2011, pp. 5-10.

[24] G. Madasamy Raja and V. Sadasivam, "Optimized Local Ternary Patterns: A New Texture Model with Set of Optimal Patterns for Texture Analysis," Journal of Computer Science, vol. 9, no. 1, Jan 2013, pp. 1-14

[25] T. Ojala, M. Pietikainen and D. Harwood, "A comparative study of texture measures with classification based on feature distributions", Pattern Recognition, vol. 29, no. 1, Jan 1996, pp. 51-59.

[26] P. Brodatz, Textures, A Photographic Album for Artists and Designers, Dover Publications, New York, 1966. 